\title{
SEVERAL REMARKS ON THE ROLE OF CERTAIN POSITIONAL AND SOCIAL GAMES IN THE CREATION OF THE SELECTED STATISTICAL AND ECONOMIC APPLICATIONS
}

\author{
Ewa DRABIK
}

\author{
Warsaw University of Technology, Faculty of Management, Warsaw, Poland \\ e-mail: ewa.drabik@poczta.fm
}

\begin{abstract}
The game theory was created on the basis of social as well as gambling games, such as chess, poker, baccarat, hex, or one-armed bandit. The aforementioned games lay solid foundations for analogous mathematical models (e.g., hex), artificial intelligence algorithms (hex), theoretical analysis of computational complexity attributable to various numerical problems (baccarat), as well as illustration of several economic dilemmas - particularly in the case where the winner takes everything (e.g., noughts and crosses). A certain gambling games, such as a horse racing, may be successfully applied to verify a wide spectrum of market mechanism, for example, market effectiveness or customer behavior in light of incoming information regarding a specific product. One of a lot applications of the slot machine (one-armed bandit) is asymptotically efficient allocation rule, which was assigned by T.L. Lai and H. Robbins (1985). In the next years, the rule was developed by another and was named a multi-armed. The aim of the paper is to discuss these social games along with their potential mathematical models, which are governed by the rules predominantly applicable to the social and natural sciences.
\end{abstract}

Key words: positional games, social games, gambling games, one-armed bandit, chess.

\section{1}

Introduction

The humanity has been accompanied by gambling and gambling games for ages, as they entertained people, provoked excitement, and gave an illusory hope for the better life. According to a representative number of scientists, the behavior of gamblers very often reflects the conduct of market players. In fact, gambling may be defined as a permanent pursuit of win, which in itself eventually becomes the prize. The decision-making process under high-risk circumstances pushes the arguments of rationality to the background. The fact in question has been included in the modern finance theory, for example, the prospect theory developed by Daniel Kahneman and Amos Tversky. The attention was particularly drawn to the market players who - while making decisions under risk - were more prone to follow herd and suffer from panic attacks. Their behavior very often proves to be irrational. In the ensuing years, the problems revolving around the game theory were becoming more and more complex. In order to provide a reasonable solution as well as to describe a sequence of social and economic phenomena, a variety of new games such as a prisoner's dilemma (originally framed by Merrill Flood and
Melvin Dresher working at RAND in 1950), gender discrimination game, chicken game, or Colonel Blotto game were created.

This paper aims to address that social games and gambling played a key role in the development of the game theory, statistics, and their applications.

\section{Some remarks about positional games}

The overwhelming majority of dilemmas related to the perfect information (PI) games were defined in the period from 1935 to 1941 and incorporated into the so-called Scottish Book. The Scottish Book referred to a notebook purchased by a wife of Stefan Banach and used by mathematicians of the Lwów School of Mathematics (such as Stanisław Mazur, Stanisław Ulam, and Hugo Steinhaus) for jotting down mathematical problems meant to be solved. The Scottish Book used to be applied for almost six years. Many problems presented therein were created in previous years and not all of them were solved. After the World War II, Lucja Banach brought the Book to Wrocław, where it was handwritten by $\mathrm{Hu}-$ go Steinhaus and sent in 1956 to Los Alamos (USA) to Stanisław Ulam. Ulam translated it into English, 
copied at his own expense, and dispatched to a variety of universities. The book in question proved to enjoy such a great popularity that it was soon published and edited - mainly in English (Duda, 2010; Mauldin, 1981).

Formally, we can write PI games as follows.

Let A be the set of strategies of player I and B be the set of strategies of player II.

$$
\varphi: \mathrm{A} \times \mathrm{B} \rightarrow \overline{\mathfrak{R}},
$$

where

$$
\bar{\Re}=\mathfrak{R} \cup\{-\infty,+\infty\}
$$

( $\mathfrak{R}$ is the set of real numbers).

This game is played as follows:

Player I chooses $a \in A$, and player II chooses $b \in B$ Both choices are made independently and without any knowledge about the choice of the other player. Then player II pays to player I the value $\varphi(a, b)$. If $\varphi(a, b)<0$ it means that player II gets from player I the value $|\varphi(a, b)|$.

The following is the idea of an infinite game of PI (perfect information):

- let $\omega \in\{0,1,2, \ldots\}$,

- there is a set $\mathrm{P}$ called the set of choices,

- player I chooses $\mathrm{p}_{0} \in \mathrm{P}$, next player II chooses $\mathrm{p}_{1} \in \mathrm{P}$, then player I chooses $\mathrm{p}_{2} \in \mathrm{P}$, and so on.

There is a function $\mathrm{f}: \mathrm{P}^{\omega} \rightarrow \bar{\Re}$ such that the end player II pays to player I the value $f\left(p_{0}, p_{1}, \ldots\right)$.

Definition 1:

The triple $\langle A, B, \varphi\rangle$ is said to be the PI-game if there exists a set $\mathrm{P}$ such that $\mathrm{A}$ is set of all function:

$$
\begin{aligned}
& \mathrm{A}=\left\{\mathrm{a}: \bigcup_{\mathrm{n}<\omega} \mathrm{P}^{\mathrm{n}} \rightarrow \mathrm{P}\right\}, \text { where } \mathrm{P}^{0}=\{\phi\}, \\
& \mathrm{B}=\left\{\mathrm{b}: \bigcup_{0<\mathrm{n}<\omega} \mathrm{P}^{\mathrm{n}} \rightarrow \mathrm{P}\right\}
\end{aligned}
$$

and there exists a function

$$
\mathrm{f}: \mathrm{P}^{\omega} \rightarrow \overline{\mathfrak{R}} \text { such that } \varphi(\mathrm{a}, \mathrm{b})=\mathrm{f}\left(\mathrm{p}_{0}, \mathrm{p}_{1}, \ldots\right),
$$

where

$$
\begin{array}{lll}
\mathrm{p}_{0}=\mathrm{a}(\varnothing), & \mathrm{p}_{1}=\mathrm{b}\left(\mathrm{p}_{0}\right), & \mathrm{p}_{2}=\mathrm{a}\left(\mathrm{p}_{1}\right), \\
\mathrm{p}_{3}=\mathrm{b}\left(\mathrm{p}_{0}, \mathrm{p}_{2}\right), & \mathrm{p}_{4}=\mathrm{a}\left(\mathrm{p}_{1}, \mathrm{p}_{3}\right)
\end{array}
$$

Definition 2:

A game $\langle A, B, \varphi\rangle$ is called determined if

$$
\inf _{b \in B} \sup _{a \in A} \varphi(a, b)=v=\sup _{a \in A} \inf _{b \in B} \varphi(a, b)
$$

Remark: A game is determined if and only if the game has a value.

A game is not determined if

$$
\inf _{b \in B} \sup _{a \in A} \varphi(a, b)<v<\operatorname{supinf}_{a \in A} \varphi(a, b)
$$

Note. If the game is not determined, then the lefthand side of (1) is larger than the right-hand side of (1).

If the game has a value $v$ and there exists an $a_{0}$ such that $\varphi\left(\mathrm{a}_{0}, \mathrm{~b}\right) \geq \mathrm{v}$ for all $\mathrm{b}$, then $\mathrm{a}_{0}$ is called an optimal strategy for player I. If $\varphi\left(a, b_{0}\right) \leq v$ for all $a$, the $b_{0}$ is called an optimal strategy for player II.

We will say that $\left\langle\mathrm{P}^{\omega}, \mathrm{f}\right\rangle$ is a win for player I or a win for the player II if $\left\langle\mathrm{P}^{\omega}, \mathrm{f}\right\rangle$ has value 1 or 0 , respectively. If $\mathrm{f}: \mathrm{P}^{\omega} \rightarrow \overline{\mathfrak{R}}$ has the property that there exists an $n$ such that $\mathrm{f}\left(\mathrm{p}_{0}, \mathrm{p}_{1} \ldots\right)$ does not depend on the choice $\mathrm{p}_{\mathrm{i}}$ with $\mathrm{i}<\mathrm{n}$, then $\left\langle\mathrm{P}^{\omega}, \mathrm{f}\right\rangle$ is called a finite game.

The following theorem is true.

Theorem 1:

Every finite game has a value.

Proof (Mycielski, 1992).

\section{The game of Hex and some infinity games with perfect information}

One of the simplest finite PI-games is Hex. Hex is played as follows.

We use a board with a 'honeycomb' with 122 cells, and we use 61 white and 61 black pieces (Fig. 1). 


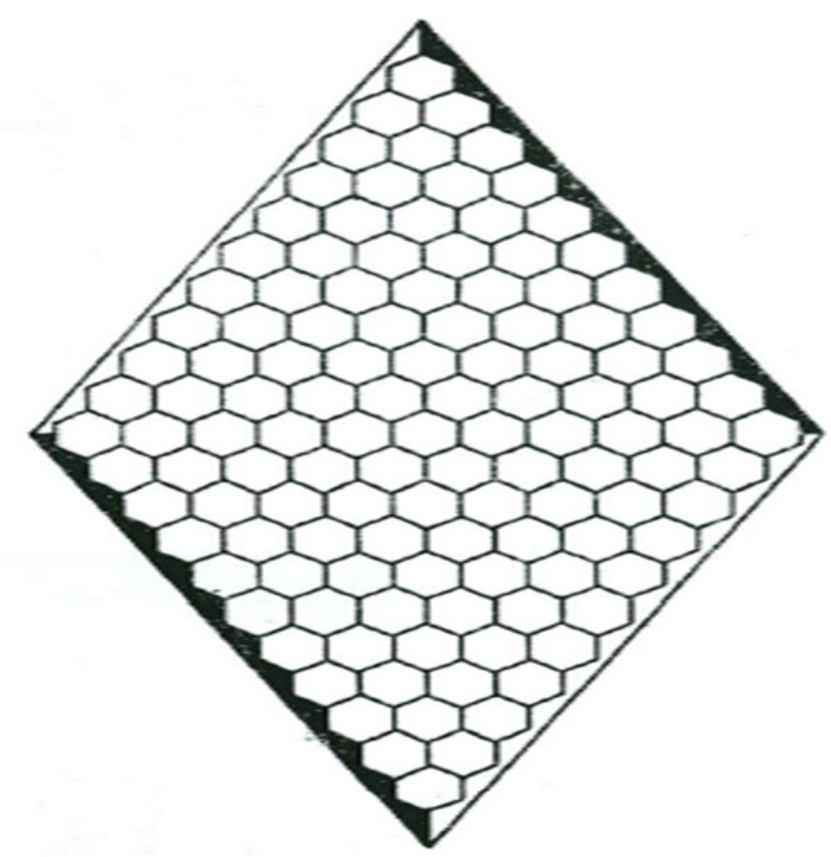

Figure 1. The board of the hex (source: Pijanowski, 1972)

The players alternatively put white or black stones on the hexagon board. White begins and he/she wins if the white stones connect the top of the board with the bottom. Black wins if the black stones connect the left edge with the right edge.

\section{Theorem 2:}

(i) When the board is filled with stones, then one of the players has won and the other has lost.

(ii) White has a winning strategy.

Proof (Mycielski, 1992).

Hex has a relative called Bridge it. But for Bridge_it, a useful description of winning strategy for player I (white) has been used. However, this does not seem to solve for the problem on hex.

Some games of the some type are difficulty compared in the sense that the problem of deciding if a position is a win for player I or for player II is complete (see Theorem 1).

The more general games are the infinity games with PI.

Let $G$ be a finite bipartite-oriented graph. In the other words, $G$ is a system $(\mathrm{P}, \mathrm{Q}, \mathrm{I})$ where $\mathrm{P}$ and $\mathrm{Q}$ are finite disjoint sets and $\mathrm{E} \subseteq((\mathrm{P} \times \mathrm{Q}) \cup(\mathrm{Q} \times \mathrm{P}))$ and $\mathrm{I}$ be set of arrows. We assume, moreover, that for each $(a, b) \in E$, there exists $c$ such that $(b, c) \in E$.
A function $\varphi: \mathrm{E} \rightarrow \mathrm{R}$ is given, and a point $\mathrm{p}_{\text {first }} \subseteq \mathrm{P}$ is fixed. The player I and player II pick alternately $\mathrm{p}_{0}=\mathrm{p}_{\text {first }}, \mathrm{q}_{0} \in \mathrm{Q}, \mathrm{p}_{1} \in \mathrm{P}, \mathrm{q}_{1} \in \mathrm{Q}, \quad \ldots \quad$ such that $\left(p_{i}, q_{i}\right) \in E$ and $\left(q_{i}, p_{i+1}\right) \in E$, thereby defining a zigzag path composed of arrows. We define three PI-games.

$\mathrm{G}_{1}$ : player II pays to player I the value

$$
\limsup _{n \rightarrow \infty} \frac{1}{2 n} \sum_{i=0}^{n-1}\left(\varphi\left(p_{i}, q_{i}\right)+\varphi\left(q_{i}, p_{i+1}\right)\right)
$$

$\mathrm{G}_{2}$ : player II pays to player I the value

$$
\liminf _{n \rightarrow \infty} \frac{1}{2 n} \sum_{i=0}^{n-1}\left(\varphi\left(p_{i}, q_{i}\right)+\varphi\left(q_{i}, p_{i+1}\right)\right)
$$

$\mathrm{G}_{3}$ : the game ends as soon as a closed loop arises in the path defined by the players, that is, as soon as player I picks any $\mathrm{p}_{\mathrm{n}} \in\left\{\mathrm{p}_{0}, . ., \mathrm{p}_{\mathrm{n}-1}\right\}$ or player II picks any $\mathrm{q}_{\mathrm{n}} \in\left\{\mathrm{q}_{0}, . ., \mathrm{q}_{\mathrm{n}-1}\right\}$.

Then player II pays to player I the 'loop average' $\mathrm{v}$ defined as follows. In the first case, $\mathrm{p}_{\mathrm{n}}=\mathrm{p}_{\mathrm{m}}$ for some $\mathrm{m}<\mathrm{n}$ and then

$$
\mathrm{v}=\frac{1}{2(\mathrm{n}-\mathrm{m})} \sum_{\mathrm{i}=0}^{\mathrm{n}-1}\left(\varphi\left(\mathrm{q}_{\mathrm{i}}, \mathrm{p}_{\mathrm{i}+1}\right)+\varphi\left(\mathrm{p}_{\mathrm{i}+1}, \mathrm{q}_{\mathrm{i}+1}\right)\right)
$$


Thus, in all three games, the players are competing to minimize or maximize the means for some numbers they encounter on the arrows of the graph. As the game $\mathrm{G}_{3}$ is finite, by Theorem 1 , it has value $v$. The infinite games $\mathrm{G}_{1}$ and $\mathrm{G}_{2}$ are determined, because they have the same value as $\mathrm{G}_{3}$. So there is at least one example where infinite PI-games help us to analyze some finite PI-games. But, some infinite PI-games are not so simple.

Example (the chase): A dog tries to catch a hare in an unbounded plane. The purpose of the dog is to minimize the time of the game and the purpose of the hare is to maximize it. We assume that each dog is farther than the hare and that only the velocities are bounded while acceleration are not. There are neat solution of those game: at each moment $t$, the hare should run full speed toward any point $a_{t}$ such that $a_{t}$ is the distant from him among all points that he can reach prior to the dog. The dog at each instant $t$ should run full speed toward that the same point $a_{t}$. The hare does not have to change the point $a_{t}$ during the game.

\section{Casino baccarat chemin de fer as a bimatrix game}

The game of baccarat played a key role in the development of game theory. Bertrand's 1889 analysis of whether player should draw or stand on a two cards total of five was the starting point of Borel investigation of strategic games. In 1924, Borel described Bertrand's study. In 1928, J. von Neumann, after providing minimax theorem, remarked that he would analyze baccarat in a paper. But a solution of the game would have to wait until the drawn of the computer age.

Baccarat is a card game played in casinos. There are three popular variants of the game: punto banco (or "North American baccarat"), baccarat chemin de fer, and baccarat banque. In baccarat chemin de fer, both players can make choice: player and banker. Each baccarat coup has three possible outcomes: "player," "banker," or "tie." Cards that have a point value of 2-9 are worth face value (in points); $10 \mathrm{~s}$, Js, Qs, and Ks have no point (i.e., are worth zero). Aces are worth 1 point. Hands are valued according to the rightmost digit of the sum of their consistent cards: for example, a card consisting of 2 and 3 is worth 5 , but a hand consisting of 6 and 7 is worth 3 (i.e., the 3 being right digit in combined points total 13 or $6+7$ $=3(\bmod 10))$. Logically then, the highest possible hand value in baccarat is 9 . The baccarat refers to anything with a value zero, in a hand of $K, 4$ and 6 .

The object of the game is to have the higher total (closer 9) at the end of the play. One player is designed as the banker who deals four cards face down: two to himself and two to the player. They look at their cards; if either has an eight or nine, this is immediately announced and the hands are turned face up and compared. If neither hand is an eight nor nine, the player has a choice to accept or refuse a third card; if accepted it is dealt face up.

Traditional practice dictates that one always accept a card if one's hand total is between 0 and 4 and always refuse a card if one's hand total is 6 or 7 . The player makes his/her decision either to accept or to refuse another card. Once both the banker and the player have made their decision, the hands are turned face up and compared. If the player's hand exceeds the banker's hand, each wagering player receives back their wager and a matching amount from the bank, and the position of banker passes. If the banker's hand exceeds the player's hand, all wagers are forfeit and placed into the bank. The banker position does not change. If there is a tie, wagers remain as they are for the next hand. A win for players pays even money. Again, hands of equal value result in a push. In the laws of baccarat, no one code is accepted as authoritative, different clubs make their own rules.

The probability that a two-card hand has a value of 0 is

$$
\left(\frac{4}{13}\right)^{2}+9\left(\frac{1}{13}\right)^{2}=\frac{25}{169}
$$

The probability that two-card hand has value $i \in\{1,2, \ldots, 9\}$ is:

$$
\frac{4}{13} \cdot \frac{1}{13}+\frac{1}{13} \cdot \frac{4}{13}+8\left(\frac{1}{13}\right)^{2}=\frac{16}{169}
$$

Player's strategy is restricted. He/she must draw on a two-card total of 4 or less and should on a twocard total of 6 or 7 . When his/her two-card total is 5 , he/she is free to draw or stand as he/she chooses. 
The decision is usually made by the player with the largest bet. Player has two pure strategies, stand on a two-card total of 5 or draw a two-card total of 5. Let $\mathrm{X}$ denote the value of players' two-card hand, and let $\mathrm{Y}$ denote the value of banker's two-card hand. Banker has a stand or draw decision in each 88 strategic situations. Another word, the pair (X, Y) is a typical bimatrix game, which has $2 \times 2^{88}$ strategies (Ethier, 2010). Ethier described some algorithms to show some strategy in Nash equilibrium for baccarat bimatrix game (see Ethier, 2010).

The algorithms based on baccarat game may be fulfilled in several manners, for example, through tax decrease, state pension increments, system of incentives such as discounts or guarantees conferred upon companies for private and public investments, road construction, and community objects developments. In the absence of simplifying assumptions, finding Nash equilibrium in the original game baccarat chemin de fer requires sophisticated algorithms, featured by a high level of computational complexity. This algorithm demands an in-depth analysis of numerous case studies. Baccarat, similar to chess, laid foundations for the development of artificial intelligence.

\section{Asymptotically efficient allocation rules and one-armed bandit}

One-armed bandit is a mechanical or an electrical machine, most frequently equipped with three drums holding a variety of card faces, used for gambling purposes. This simple machine has been known since 1887, when Charles Fey devised an automatic mechanism to make the trade of offered products more attractive. Since the machines proved to be very successful, in the middle of the 20th century, the mechanical constructions were substituted by computerized devices that were used in casinos. They used to entertain and divert the ladies whose husbands and fiancés devoted themselves to card games.

First machines worked on a fully random basis. Currently, because of the applicable software, the randomness is of partial nature. The participation in such games and generation of shots attracted the attention of many scientists who predominantly aimed to elaborate on profit-maximizing strategies. The strategies concerned became attributable to many disciplines, such as economy, biology, and steering theory (compare the available literature [4] or [1]).

Unstated but implicit in the definition was requirement that the players insert one or more coins into slot (hence, the name "slot machine") in order to activate the machine. Now, almost all slots machines are electronic and controlled by microprocessors. The microprocessor is programmed a randomnumber generator that operates continually. The moment the player pulls the handle or presses the button to activate the machine, the most recently generated random numbers determine the outcome almost instantly. Despite all this, the underlying mathematics of the act of the modern slot machine much different from that of classical machine.

The slot machines find a lot of applications, for instance, in statistics. One of them is asymptotically efficient allocation adaptive rule, which was assigned by Lai and Robbins (1985). In the next years, the rule was developed by other scientists and it was named multi-armed bandit problem.

One of the strategies - the asymptotically efficient adaptive allocation rule - that is used in the realm of statistics may be described as follows.

Let $\mathrm{w}_{\mathrm{i}}(\mathrm{i}=1,2, \ldots)$ denote statistical populations (treatments, manufacturing processes, etc.) specified by univariate (or another) density function $\mathrm{f}\left(\mathrm{w}, \theta_{\mathrm{i}}\right)$ with respect measure $v$, where $f(.$, .) is known parameter and $\theta_{\mathrm{i}}$ is unknown parameter belonging to some set $\Theta$. Assume that

$$
\int_{-\infty}^{\infty}|w| f(w, \theta) d v(w)<\infty \text { for all } \theta \in \Theta
$$

We sample $\mathrm{w}_{1}, \mathrm{w}_{2}, \ldots$ sequentially from the $\mathrm{k}$ populations in order to achieve the greatest possible expected value $S_{n}=w_{1}+\ldots, w_{n}$ as $n \rightarrow \infty$, where $n$ is number of observations. The multi-armed bandits problem derives from an imagined slot machine with $\mathrm{k} \geq 2$ arms. When an arm is pulled, the player wins a random reward. For each arm $\mathrm{j}$, there is an unknown probability distribution of the reward. The player wants to choose depending in some way on the reward of previous trials, so as to maximize the long-run total expected reward. 
An adaptive allocation rule $\varphi$ is a sequence of random variables $\varphi_{1}, \varphi_{2}, \ldots$ taking values in the set $\{1$, $\ldots, \mathrm{k}\}$ and such that the $\varphi_{\mathrm{t}}=0$ when player don't play (sample) or $\varphi_{\mathrm{t}}=1$ when player play. The expected reward is

$$
\mu(\theta)=\int_{-\infty}^{+\infty} \mathrm{wf}(\mathrm{w} ; \theta) \mathrm{d} v(\mathrm{w})
$$

Another way, the expected reward is

$$
\mathrm{ES}_{\mathrm{n}}=\sum_{\mathrm{j}=0}^{\mathrm{k}} \sum_{\mathrm{i}=1}^{\mathrm{n}} \mathrm{E}\left(\mathrm{w}_{\mathrm{i}} \mathrm{I}_{\left\{\varphi_{\mathrm{i}}=\mathrm{j}\right\}} \mid \mathfrak{I}_{\mathrm{i}-1}\right)=\sum_{\mathrm{j}=0}^{\mathrm{k}} \mu(\theta) \mathrm{T}_{\mathrm{n}}(\mathrm{j})
$$

where:

$\mathrm{ES}_{\mathrm{n}}$ is conditional expected value of regret,

$T_{n}(j)=\sum_{i=1}^{n} I_{\left\{\varphi_{i}=j\right\}}$ is the number of times that $\varphi$ samples to stage $\mathrm{n}$. The problem of maximizing $\mathrm{ES}_{\mathrm{n}}$ is, therefore, equivalent to that of minimizing to regret

$$
\begin{aligned}
& \mathrm{R}_{\mathrm{n}}\left(\theta \theta=\mathrm{n} \mu^{*}-\mathrm{ES}_{\mathrm{n}}=\right. \\
& \sum_{\mathrm{j}: \mu\left(\theta_{\mathrm{j}}\right)<\mu^{*}}\left(\mu^{*}-\mu\left(\theta_{\mathrm{j}}\right)\right) \mathrm{ET}_{\mathrm{n}}(\mathrm{j})
\end{aligned}
$$

where:

$$
\mu^{*}=\max \left\{\mu\left(\theta_{0}\right), \mu\left(\theta_{1}\right)\right\}=\mu\left(\theta^{*}\right)
$$

for some

$$
\theta^{*} \in\left\{\theta_{0}, \theta_{1}\right\}
$$

Let $\mathrm{I}(\theta, \lambda)$ denote the Kullback-Leibler number

$$
\mathrm{I}\left(\theta(\lambda)=\int_{-\infty}^{\infty} \log \frac{\mathrm{f}(\mathrm{w} ; \theta)}{\mathrm{f}(\mathrm{w} ; \lambda)} \cdot \mathrm{f}(\mathrm{w} ; \theta) \mathrm{dv}(\mathrm{w})\right.
$$

such that $0<\mathrm{I}(\theta, \lambda)<\infty$ whenever $\mu(\lambda)>\mu(\theta)$.

Generally, regret is

$$
\mathrm{R}_{\mathrm{n}}(\theta) \approx\left\{\sum_{\mathrm{j}: \mu\left(\theta_{\mathrm{j}}\right)<\mu^{*}}\left(\mu^{*}-\mu\left(\theta_{\mathrm{j}}\right)\right) / \mathrm{I}\left(\theta_{\mathrm{j}}, \theta^{*}\right)\right\} \log \mathrm{n},
$$

The rule $\varphi$ :

Let $\mathrm{w}_{1}, \mathrm{w}_{2}, \ldots$ be a sequence of random variables whose distribution is parameterized by unknown parameter $\theta \in \Theta$.
Let $\mathrm{g}_{\mathrm{nt}}: \mathfrak{R}^{\mathrm{t}} \rightarrow \mathfrak{R}(\mathrm{n}=1,2, \ldots ; \mathrm{t}=1, \ldots, \mathrm{n})$ be the Borel function such that for every $\theta \in \Theta$,

(W1)

$$
\mathrm{P}_{\theta}\left\{\mathrm{r} \leq \mathrm{g}_{\mathrm{nt}}\left(\mathrm{w}_{1}, \ldots, \mathrm{w}_{\mathrm{t}}\right) \quad \text { for all } \mathrm{t} \leq \mathrm{n}\right\}=1-\mathrm{o}\left(\mathrm{n}^{-1}\right)
$$

$$
\begin{gathered}
\lim _{\varepsilon \rightarrow 0}\left(\underset{\substack{\mathrm{n} \rightarrow \infty \\
\limsup }}{\mathrm{n}} \sum_{\mathrm{t}=1} \mathrm{P}_{\theta}\left\{\mathrm{g}_{\mathrm{nt}}\left(\mathrm{w}_{1}, \ldots, \mathrm{w}_{\mathrm{t}}\right) \geq \mu(\lambda)-\varepsilon\right\} / \log \mathrm{n}\right) \\
\leq 1 / \mathrm{I}(\theta / \lambda)
\end{gathered}
$$

whenever $\mu(\lambda)>\mu(\theta)$.

(W3)

$\mathrm{g}_{\mathrm{nt}}$ is nondecreasing in $\mathrm{n} \geq \mathrm{t}$ for every fixed $\mathrm{t}=1,2, \ldots$

Let $\mathrm{h}_{\mathrm{t}}\left(\mathrm{w}_{1}, \ldots, \mathrm{w}_{\mathrm{t}}\right)$ be the Borel function, $\mathrm{h}_{\mathrm{t}}: \mathfrak{R}^{\mathrm{t}} \rightarrow \mathfrak{R}$, such that for every $\theta \in \Theta$

$\mathrm{h}_{\mathrm{t}} \leq \mathrm{g}_{\mathrm{nt}}$ for all $\theta \in \Theta$

(W5)

$$
\mathrm{P}_{\theta}\left\{\max _{\mathrm{t}}\left|\mathrm{h}_{\mathrm{t}}\left(\mathrm{w}_{1}, \ldots, \mathrm{w}_{\mathrm{t}}\right)-\mu(\theta)\right|>\varepsilon\right\}=\mathrm{o}\left(\mathrm{n}^{-1}\right)
$$

for every $\varepsilon>0$.

Condition W5 can be satisfied for the average:

$$
\mathrm{h}_{\mathrm{t}}\left(\mathrm{w}_{1}, \ldots, \mathrm{w}_{\mathrm{t}}\right)=\left(\mathrm{w}_{1}+\ldots+\mathrm{w}_{\mathrm{t}}\right) / \mathrm{t}
$$

if

$$
\mathrm{E}_{\theta} \mathrm{w}_{\mathrm{i}}^{2}<\infty \quad(\mathrm{i}=1, \ldots, \mathrm{t})
$$

and

$$
\mathrm{g}_{\mathrm{nt}}\left(\mathrm{w}_{1} \ldots, \mathrm{w}_{\mathrm{t}}\right)=\overline{\mathrm{w}_{\mathrm{t}}}+\sigma\left(2 \mathrm{a}_{\mathrm{nt}}\right)^{1 / 2} \text { for } \mathrm{n} \geq \mathrm{t}
$$

where:

$\sigma$ is the standard deviation, $a_{n t}(n=1,2, \ldots ; t=1, \ldots, n)$ is positive constant such that for all $t, a_{n t}$ is nondecreasing for all $n \geq t$, and there exists $\varepsilon \rightarrow 0$ such that

$$
\left|\mathrm{a}_{\mathrm{nt}}-\frac{\log \mathrm{n}}{\mathrm{t}}\right| \leq \frac{\varepsilon(\log n)^{1 / 2}}{\mathrm{t}^{1 / 2}} \text { for all } \mathrm{t} \leq \mathrm{n}
$$

Remark: If

$$
f(w ; \theta)=\left(2 \pi \sigma^{2}\right)^{-1 / 2} \exp \left\{-\frac{(w-\theta)^{2}}{2 \sigma^{2}}\right\},
$$

then

$$
\mathrm{I}\left(\theta(\lambda)=\frac{(\theta-\lambda)^{2}}{2 \sigma^{2}}\right.
$$


Let $\mathrm{w}_{1}, \ldots, \mathrm{w}_{\mathrm{T}_{\mathrm{n}}(\mathrm{j})}$ be the successive rewards obtained from arm $\mathrm{j}$ up to stage $\mathrm{n}$. The upper confidence bound $U_{n}(j)$ and the point estimate $\mu_{n}(j)$, the mean reward under arm $\mathrm{j}$, are given by

$$
\begin{aligned}
& \mu_{n}(j)=h_{T_{n}(j)}\left(w_{1}, \ldots, w_{T_{n}(j)}\right) \\
& U_{n}(j)=g_{n, T_{n}(j)}\left(w_{1}, \ldots, w_{T_{n}(j)}\right) .
\end{aligned}
$$

Define the "leader" at stage $\mathrm{n}$ as the population with the largest estimated mean among all population. The following rule $\varphi$ decides which arms to play.

In the first stage, we observe $\mathrm{w}_{1}, \mathrm{w}_{2}, \ldots, \mathrm{w}_{\mathrm{n}}$ and compute $U_{n}(j)$ and $\mu_{n}(j)$. Next we use one of the following decisions:

1) If arm $j$ is already one of m-leaders choose to play the m-leaders,

2) If $\operatorname{arm} j$ is not among the m-leaders and $U_{n}(j)$ is less than $\mu_{\mathrm{n}}(\mathrm{k})$ for every m-leader $\mathrm{k}$, then again the m-leaders play.

3) If arm $j$ is not among the m-leaders and $\mu n \leq$ Un of least best of m-leaders, then play the $(m-1)-$ best of m-leaders and arm $\mathrm{j}$.

Note in any case, the $(m-1)$ of m-leaders always get played.

Theorem 3:

The rule $\varphi$ jest is asymptotically efficient.

Proof (Ananatharam V., Varaiya P., and Warland J., 1987).

\section{Some remarks about chess}

The most common "finite" positional game with PI is chess, which laid foundations for artificial intelligence algorithms applied in various domains, including the construction of dynamic equilibrium models as well as the description of economic systems lacking the equilibrium.

The artificial intelligence is more and more often applied in energetic - to create systems not only monitoring the course of specific processes but also involved in planning and decision-making procedures. It is used also for the purposes of image processing, for example, in cameras, supporting financial decisions, as well as many other domains of everyday life.
It is worth mentioning one of the most fascinating personalities of sports and science, Robert James Fischer who was famous for his exceptionally talented and rebellious mind. He played hundreds of outstanding games, implemented many innovative solutions, and introduced the so-called Fischer clock enabling to keep track of the total time each player takes for his or her own moves. Owing to some personal reasons, he was not able to play the game with the computer. Just wonder who would have won in such a competition.

In the light of the game theory, it should be emphasized that because of its limited range of strategies, the chess game is indeterminate, of which fact the vast majority of chess players remain unaware.

\section{$7 \quad$ Summary and remarks}

It is worth noting that certain gambling games, such as horse racing, may be successfully applied to verify a wide spectrum of market mechanisms, for example, market effectiveness, or consumer behavior in light of incoming information regarding a specific product.

The methods of reasoning based on the game theory are used for examining the equilibrium states in a diversity of economic phenomena. In this case, the suboptimal equilibrium (i.e., the best state for all individual players) is replaced by the general equilibrium.

The situation concerned occurs whenever particular players abstain from the available, yet globally undesirable, ways of conduct (e.g., through subsidizing the investments that for particular players seem unjustifiable and unreasonable or consumption that is not necessary for the attainment of global equilibrium corresponding to the notion of Nash equilibrium).

Certainly, since Keynes's times the developers of economic politics have attempted to move the economic equilibrium towards the production increase and, analogous, towards the growth of demand determining the shape of manufacturing system that would be maintainable without an unreasonable accumulation of potential reserves. 


\section{$8 \quad$ References}

[1] Ananatharam, V., Varaiya, P., Warland, J., 1987. Asymptotically Efficient Allocation Rules for the Multiarmed Bandit Problem with Multiple Plays - Part I: I.I.D. Rewards. IEEE Transaction of Automatic Control, Vol. Ac-32, No. 11, pp.968-976.

[2] Duda, R., 2010. Lwow School of Mathematics. Wroclaw: Wroclaw University Publishing House.

[3] Ethier, S.N., 2010. The Doctrine of Chances: Probabilistic Aspects of Gambling. Berlin Heidelberg: Springer Verlag.
[4] Lai, T.L., Robbins, H., 1985. Asymptotically Efficient Adaptive Allocation Rules. Advanced in Applied Mathematics, Vol. 6, pp.4-22.

[5] Mauldin, R.D., 1981. The Scottish Book. Mathematics from the Scottish Café. Boston - Basel Stuttgart: Birkhausen.

[6] Mycielski, J., 1992. Games with Perfect Information. In: R.J. Aumann, S. Hart (eds.). Handbook of Game theory with Economic Application, Vol. 1, North - Holland, Amsterdam, pp.20-40.

[7] Pijanowski, L., 1972. Przewodnik gier (Game Guide). Warszawa: Iskry. 